The trial enrolled 351 patients aged $\geq 50$ years with International Prostate Symptom Score (IPSS) $\geq 13$, maximum urinary flow rate $5-15 \mathrm{ml} / \mathrm{s}$, total prostate volume $\geq 30 \mathrm{ml}$, postvoid residual urine volume $\leq 200 \mathrm{ml}$ and fasting serum LDL 100-190 mg/dl. After a 2-week run-in period with placebo, patients were randomly allocated to receive either $80 \mathrm{mg}$ atorvastatin or placebo once daily for 26 weeks, with efficacy evaluations performed at screening, baseline, and weeks 4, 13 and 26.

Overall, 319 patients completed the study (treatment group $n=160$, placebo group $n=159$ ). After 26 weeks, there were no significant differences between the atorvastatin group and the placebo group for changes in primary or secondary LUTS outcome measures, including IPSS, total prostate volume, maximum urinary flow rate and PSA. There were significantly greater reductions in the levels of serum lipid markers in the atorvastatin group compared with placebo, including LDL and triglycerides.

The authors conclude that atorvastatin, at a dose of $80 \mathrm{mg}$ daily, is ineffective for treating LUTS in men with prostatic enlargement and $\mathrm{LDL}<190 \mathrm{mg} / \mathrm{dl}$.

Original article Mills IW et al. (2007) Atorvastatin treatment for men with lower urinary tract symptoms and benign prostatic enlargement. Eur Urol 52: 503-509

\section{Comparison of high-frequency jet ventilation and spontaneous ventilation in ESWL procedures}

A variety of anesthetic techniques can be used when performing extracorporeal shock wave lithotripsy (ESWL), although spontaneous ventilation with a laryngeal mask airway has become a common approach. High-frequency jet ventilation (HFJV) is thought to reduce stone movement and time and shock energy requirements, therefore decreasing exposure to $\mathrm{X}$-rays and perinephric trauma. Cormack and colleagues retrospectively compared spontaneous ventilation with HFJV in patients who underwent ESWL.

The study included data from 91 consecutive patients who were anesthetized with either spontaneous ventilation $(n=39)$ or HFJV $(n=52)$ during ESWL between October 2002 and February 2004. Both groups were matched for age, sex, weight and calculus position (renal or ureteric). HFJV was performed via a Ben Jet tube (Baldwin Medical, Knoxfield, Victoria, Australia) at $150-250$ breaths per minute with an oropharyngeal airway. The airway was maintained using a laryngeal mask in the spontaneous ventilation group.

Significantly fewer shocks were required in the HFJV group than the spontaneous ventilation group (median 3,000 vs 2,$000 ; P=0.01$ ), and the median total shock power was significantly lower in the HFJV group compared with the spontaneous ventilation group $(P=0.001)$. The rate of postoperative complications was low and similar between groups, and postoperative X-ray showed similar fragmentation rates.

The authors conclude that HFJV is an effective anesthetic approach for ESWL and, as it requires a lower shock energy and number of shocks than spontaneous ventilation, might reduce perinephric trauma and pain.

Original article Cormack JR et al. (2007) Comparison of two ventilation techniques during general anesthesia for extracorporeal shock wave lithotripsy: high-frequency jet ventilation versus spontaneous ventilation with a laryngeal mask airway. Urology 70: 7-10

\section{Radium-223 might benefit patients with hormone-refractory prostate cancer}

The high risk of bone involvement in cases of hormone-refractory prostate cancer has previously been treated with bisphosphonates (which reduce the risk of skeletal events but do not extend survival) or beta-emitting radioisotopes, which improve pain but have myelotoxic effects. Nilsson and colleagues conducted a small, randomized, placebo-controlled, phase II trial of the alpha-emitter radium-223 $\left({ }^{223} \mathrm{Ra}\right)$ for the treatment of these patients. A phase I trial in patients with metastatic bone disease had shown preferential uptake of ${ }^{223} \mathrm{RA}$ in skeletal metastases and that the treatment was well tolerated.

Among 64 patients with hormone-refractory prostate cancer and bone pain, who all received external beam radiotherapy at baseline, 33 were randomly assigned four intravenous injections of $50 \mathrm{kBq} / \mathrm{kg}{ }^{223} \mathrm{Ra}$ and 31 received a saline solution (placebo) at weeks 0, 4, 8 and 12. Median relative change in bone alkaline phosphatasea marker of disease extent and prognosis - was assessed 4 weeks after the last injection.

There was a significant reduction in the prognostic marker bone alkaline phosphatase in 